\author{
Augusto DegiovanNi* - Paolo MaGRINI**
}

\title{
Nuovi atti nomenclatoriali nel genere Trechus Clairville, 1806 (Coleoptera, Carabidae, Trechinae)
}

\begin{abstract}
Riassunto: Facendo seguito alla monografia, e alla relativa iconografia, del genere Trechus in Italia e Corsica di Degiovanni \& Magrini (2019), gli autori propongono questa breve nota di sintesi, con "addenda \& corrigenda", per chiarire e/o aggiornare i contenuti del testo.
\end{abstract}

Abstract: Following the monograph, and related iconography, on the genus Trechus in Italy and Corsica by Degiovanni \& Magrini (2019), the authors proposes this short summary note, with "addenda \& corrigenda", to clarify and/or update the text.

Key words: Monograph Italian Trechus, addenda, corrigenda.

\section{SINONIMIE}

In base ad un accurato esame del materiale tipico o topotipico, vengono proposte le seguenti sinonimie.

- Trechus danieli perinii Holdhaus, $1950=$ Trechus danieli danieli Holdhaus, 1902 n. syn.

- $\quad$ Trechus bensai Jeannel, 1927 = Trechus montispennae Jeannel, 1927 n. syn.

[le due specie sono state descritte nello stesso lavoro (Jeannel, 1927), rispettivamente a pg. 574 ( $T$. montispennae) ed a pg. 576 (T. bensai). Per il Principio del Primo Revisore, articoli 24.2.1 e 24.2.2 del Codice di Nomenclatura Zoologica (ICZN, 1999), stabiliamo la precedenza di Trechus montispennae Jeannel, 1927 su Trechus bensai Jeannel, 1927 , che quindi è considerato suo sinonimo più recente].

- $\quad$ Trechus dolomitanus venetus Jeannel, 1931 = Trechus dolomitanus dolomitanus Jeannel, 1931 n. syn.

[I taxa dolomitanus e venetus furono descritti da Jeannel (1931) nello stesso lavoro e nella stessa pagina (pg. 50), entrambi come sottospecie di Trechus tristiculus K. Daniel \& J. Daniel, 1898. Più tardi Schönmann (1937), agendo come Primo Revisore, articoli 24.2.1 e 24.2.2 del Codice di Nomenclatura Zoologica (ICZN, 1999), considerò dolomitanus buona specie, e venetus sua sottospe- cie. A nostro avviso i due taxa sono sinonimi e, per l'articolo 24.1 del Codice (ICZN, 1999), diamo la priorità al taxon con il rango più alto, quindi $T$. dolomitanus].

- Trechus montiscusnae Focarile, $1952=$ Trechus zangherii Jeannel, 1927 n. syn.

- Trechus obtusus lucanus Focarile, $1949=$ Trechus obtusus obtusus Erichson, 1837 n. syn.

- Trechus orobicum Monguzzi, 2015 = Trechus bergamascus Jeannel, 1927 n. syn.

\section{CAMBIO DI GRUPPO}

Si ritiene che Trechus laevipes Jeannel, 1927 non possa essere mantenuto nel gruppo pertyi, come indicava Jeannel, 1927 nella sua monografia, per la diversa conformazione principalmente del lobo mediano dell'edeago e della lamella copulatrice, che risultano invece simili a quelli delle specie del gruppo pyrenaeus (sensu Jeannel, 1927) nel quale viene pertanto inserito. Al gruppo pyrenaeus va anche ascritto Trechus sessitanus Monguzzi, 1985 per gli stessi motivi. Di conseguenza, in pieno accordo con l'amico Riccardo Monguzzi di Milano, si indicano come taxa di Italia e Corsica appartenenti al gruppo pyrenaeus le seguenti tre specie: Trechus varendorffi Sainte Claire-Deville, 1903, Trechus laevipes Jeannel, 1927 e Trechus sessitanus Monguzzi, 1985; la somiglianza dei genitali maschili di questi taxa, oltre che in visione laterale, è

\footnotetext{
*Augusto Degiovanni, Via Andrea Costa, 6, 40027 Bubano di Mordano (BO), Italia. E-mail: degiovanni1959@gmail.com **Paolo Magrini, Museo di Storia Naturale dell'Università di Firenze, Sezione di Zoologia "La Specola", Via Romana 17, 50125 Firenze, Italia. E-mail: duvalius@paolomagrini.it
} 
anche ben evidente in visione ventrale (cfr. Degiovanni \& Magrini, 2019: tav. 109-111-113).

\section{CAMBio Di STATUS}

Trechus montismaiellettae Ghidini, 1931 e Trechus angelae Magrini, 1984 sono da ritenersi buone specie, come riportato nelle descrizioni originali, in particolare per differenze costanti ed evidenti dell'organo copulatore maschile (cfr. Degiovanni \& Magrini, 2019: tav. 14 e 28) e non sottospecie di Trechus straneoi Jeannel, 1931, come indicato in Battoni \& Vigna Taglianti, 1994. A tale proposito si consideri anche la notevole distanza dei due massicci montuosi habitat dei due taxa, dall'areale delle due sottospecie, ritenute valide, di Trechus straneoi: ssp. straneoi Jeannel, 1931 e ssp. cerrutii Focarile, 1950.

Indubbiamente future indagini molecolari potranno risolvere la questione in maniera più esaustiva.

\section{ERRATA CORRIGE}

In base ad osservazioni personali e su indicazione di alcuni colleghi, in particolare Riccardo Monguzzi, apportiamo le seguenti correzioni al testo della nostra monografia (Degiovanni \& Magrini, 2019):

- Trechus montisrosae Jeannel, 1921 pag. 224, va corretta la distribuzione, non Val Sessera ma: Alta Valle Anzasca (convalle della Val d'Ossola) e Alta Val Sermenza (convalle della Valsesia): propaggini orientali del Monte Rosa.

- Trechus meschniggi Jeannel, 1930 pag. 100, la distribuzione indicata va sostituita con la seguente: Prealpi Venete [Col Nudo (BL)] e Alpi Carniche: Monte Raut (PN), Monte Teverone (PN), Val Cimoliana m 1000 s.l.m. (PN), Monfalcon di Montanaia m 1800 s.l.m., Val Cimoliana (PN); Monte Verzegnis (UD).

- Trechus salassus Jeannel, 1927 pag. 79, correggere la distribuzione indicata con la seguente: specie endemica italiana localizzata nelle Alpi Nord-occidentali: T. salassus salassus è noto delle Alpi Pennine [Monte Massone e Monte Capezzone (Alta Valstrona e Alta Valsesia)], mentre T. salassus montiszedae è noto delle Alpi Lepontine: Cima Laurasca e Monte Zeda (a nord-ovest del Lago Maggiore).

- Trechus bergamascus Jeannel, 1927 pag. 44 e 46 va corretta la seguente provincia: M. Guglielmo, Val Palot (BS).

\section{RINGRAZIAMENTI}

Ringraziamo sentitamente Stefano Ziani di Meldola (FC), per la revisione critica del testo.

\section{BIBLIOGRAFIA}

Battoni F., Vigna Taglianti A., 1994 - Osservazioni sui Trechus appenninici del gruppo obtusiusculus (Coleopterta, Carabidae). Fragmenta entomologica, Roma,25(2): 293-307.

Degiovanni A., Magrini P., 2019 - I Trechus d'Italia e Corsica. Monografie Entomologiche Vol. II., Natura Edizioni Scientifiche, 274 pp, 166 Tav.

ICZN [International Commission on Zoological Nomenclature], 1999 - International Code of Zoological Nomenclature adopted by the International Union of Biological Sciences (Fourth Edition). International Trust for Zoological Nomenclature, London, $306 \mathrm{p}$.

JEANNEL R., 1927 - Monographie des Trechinae 2. Morphologie comparée et distribution géographique d'un groupe de Coléoptères. L'Abeille, Paris, 33: 1-592.

JEANNEL R., 1931 - Trechinae et Bathysciinae nouveaux de l'Italie. Bollettino della Società entomologica italiana, 63: 49-54.

SCHÖNMANN R., 1937 - Die Artsystematik und geographische Verbreitung der hochalpinen Trechini der Ostalpen. Zoologische Jahrbücher. Abteilung für Systematik, Ökologie und Geographie der Tiere, 70: 177-226. 